\title{
POINTWISE CONVERGENCE OF TRIGONOMETRIC SERIES
}

\section{CHANG-PAO CHEN}

(Received 22 October 1985)

Communicated by W. Moran

\begin{abstract}
We establish two results in the pointwise convergence problem of a trigonometric series

$$
\sum_{|n|<\infty} c_{n} e^{i n t} \text { with } \lim _{\lambda \downarrow 1} \varlimsup_{n \rightarrow \infty} \sum_{|k|=n}^{[\lambda n]}\left|\Delta^{m} c_{k}\right|=0
$$

for some nonnegative integer $m$. These results not only generalize Hardy's theorem, the Jordan test theorem and Fatou's theorem, but also complement the results on pointwise convergence of those Fourier series associated with known $L^{1}$-convergence classes. A similar result is also established for the case that $\lim _{n \rightarrow \infty} \sum_{|k|=n}^{n+\left[n / l_{n}\right]}\left|J^{m} c_{k}\right|=0$, where $\left\{l_{n}\right\}$ satisfies certain conditions.
\end{abstract}

1980 Mathematics subject classification (Amer. Math. Soc): 42 A 20, 42 A 32.

Keywords and phrases: pointwise convergence of a trigonometric series, Fourier series.

Let $\left\{c_{n}\right\}$ be a sequence of complex numbers. Define $\Delta^{m} c_{n}(m \geqslant 0)$ by the following recursive relations: for $n>0$,

$$
\begin{aligned}
& \Delta^{0} c_{n}=c_{n}, \quad \Delta^{0} c_{-n}=c_{-n} ; \\
& \Delta c_{n}=c_{n}-c_{n+1}, \quad \Delta c_{-n}=c_{-n}-c_{-n-1} ; \\
& \Delta^{m} c_{n}=\Delta \Delta^{m-1} c_{n}, \quad \Delta^{m} c_{-n}=\Delta \Delta^{m-1} c_{-n} \quad(m>1) .
\end{aligned}
$$

We call $\Delta^{m}$ the $m$ th difference operator. It is clear that for $n>0$ and $m \geqslant 0$,

$$
\begin{aligned}
& \Delta^{m} c_{n}=c_{n}-\left(\begin{array}{c}
m \\
1
\end{array}\right) c_{n+1}+\left(\begin{array}{c}
m \\
2
\end{array}\right) c_{n+2}-\cdots+(-1)^{m}\left(\begin{array}{l}
m \\
m
\end{array}\right) c_{n+m} \\
& \Delta^{m} c_{-n}=c_{-n}-\left(\begin{array}{c}
m \\
1
\end{array}\right) c_{-n-1}+\left(\begin{array}{c}
m \\
2
\end{array}\right) c_{-n-2}-\cdots+(-1)^{m}\left(\begin{array}{c}
m \\
m
\end{array}\right) c_{-n-m} .
\end{aligned}
$$

(c) 1987 Australian Mathematical Society 0263-6115/87 \$A2.00+0.00 
In this paper, we use $S_{n}(t)$ and $\sigma_{n}(t)$ to denote the $n$th partial sum and the $n$th Cesàro sum of the trigonometric series $\sum_{|n|<\infty} c_{n} e^{i n t}$. If the series is the Fourier series of an integrable function $f$, we write $S_{n}(f, t)$ and $\sigma_{n}(f, t)$ instead of $S_{n}(t)$ and $\sigma_{n}(t)$, respectively. In [2], the $L^{1}$-convergence property of a Fourier series was discussed, and many $L^{1}$-convergence classes were established there. In this paper, the pointwise convergence property of these Fourier series will be established. Indeed, the pointwise convergence property will be established for the following more general case.

THEOREM 1. If $\left\{c_{n}\right\}$ is a null sequence of complex numbers with

$$
\lim _{\lambda \downarrow 1} \varlimsup_{n \rightarrow \infty} \sum_{|k|=n}^{[\lambda n]}\left|\Delta^{m} c_{k}\right|=0
$$

for some nonnegative integer $m$, then, for $0<\left|t_{0}\right| \leqslant \pi, S_{n}\left(t_{0}\right)$ and $\sigma_{n}\left(t_{0}\right)$ converge together to the same limit, or they both diverge. Furthermore, let $E$ be any subset of $T$ bounded away from 0 , i.e.,

$$
E \subset\{t: \delta \leqslant|t| \leqslant \pi\} \quad \text { for some } \delta>0 .
$$

Then $S_{n}(t)$ converges uniformly on $E$ if and only if $\sigma_{n}(t)$ converges uniformly on $E$. Whenever $m=0$, the conditions ' $0<\left|t_{0}\right| \leqslant \pi$ ' and ' $E$ is bounded away from 0 ' can be eliminated.

REMARK. Theorem 1 will apply to many particular cases. Before proving the theorem, let us investigate these applications. The first case we want to investigate is that in which

$$
n c_{n}=O(1) \quad(|n| \rightarrow \infty) .
$$

If condition (i) holds, then there exists an absolute constant $M$ such that

$$
\sum_{|k|=n}^{[\lambda n]}\left|c_{k}\right| \leqslant M\left(\frac{1}{n}+\log \lambda\right) \text { for all } n \geqslant 1 \text { and } \lambda>1 .
$$

The above inequality implies that condition $(\Delta)$ corresponding to $m=0$ holds. Therefore, Theorem 1 can be applied to any trigonometric series satisfying condition (i), in particular to the Fourier series of any function of bounded variation. From the above result, we see that Theorem 1 generalizes a theorem of Hardy, Theorem II.2.2 in [5].

The second case we want to investigate is the following Tauberian condition of Hardy-Karamata kind:

$$
\lim _{\lambda \downarrow 1} \varlimsup_{n \rightarrow \infty} \sum_{|k|=n}^{[\lambda n]}|k|^{p-1}\left|\Delta c_{k}\right|^{p}=0 \quad(1<p \leqslant 2)
$$


(cf. $[2,4])$. As shown in [2], case (ii) is a generalization of case (i). By the Hölder inequality, we get

$$
\sum_{|k|=n}^{[\lambda n]}\left|\Delta c_{k}\right| \leqslant(2 \lambda)^{1 / q}\left(\sum_{|k|=n}^{[\lambda n]}|k|^{p-1}\left|\Delta c_{k}\right|^{p}\right)^{1 / p},
$$

where $1 / p+1 / q=1$. From this inequality, we infer that Theorem 1 can be applied to any Fourier series satisfying condition (ii). From the following set of implications

$$
\begin{aligned}
\sum_{|k|=1}^{\infty}|k|^{p-1}\left|\Delta c_{k}\right|^{p} & <\infty \Rightarrow n\left(\frac{\sum_{|k|=n}^{\infty}\left|\Delta c_{k}\right|^{p}}{n}\right)^{1 / p}=o(1) \\
& \Rightarrow \frac{1}{n} \sum_{|k|=n}^{2 n}|k|^{p}\left|\Delta c_{k}\right|^{p}=o(1) \\
& \Rightarrow \lim _{\lambda \downarrow 1} \varlimsup_{n \rightarrow \infty} \sum_{|k|=n}^{[\lambda n]}|k|^{p-1}\left|\Delta c_{k}\right|^{p}=0,
\end{aligned}
$$

we find that the conclusions of Theorem 1 hold for each of the classes $\mathscr{C}_{p}, \mathscr{C}_{p}^{*}$ and $\mathscr{V}_{p}$, defined in $[1,7]$.

The third case we want to investigate is the following condition:

$$
\lim _{\lambda \downarrow 1} \varlimsup_{n \rightarrow \infty}(\log n) \sum_{|k|=n}^{[\lambda n]}\left|\Delta c_{k}\right|=0 .
$$

This condition is the limit of condition (ii) as $p \rightarrow 1^{+}$. It evidently implies condition $(\Delta)$.

The fourth case we want to investigate is the following condition:

$$
\sum_{|n|=1}^{\infty}\left|\Delta^{m} c_{n}\right|<\infty \quad(m \geqslant 0) .
$$

It is clear that Theorem 1 will apply to this case. The special case of (iv) corresponding to $m=1$ says that Theorem 1 also applies to $\mathscr{B} \mathscr{V}$, the class of bounded variation, defined in [2]. Let $\mathscr{C}, \mathscr{S}$ and $\mathscr{F}_{p}$ be as in [2,7]. As shown in [7], the following relations hold:

$$
\text { \{even quasi-convex null-sequences }\} \subset \mathscr{S} \subset \mathscr{F}_{p} \subset \mathscr{C} \cap \mathscr{B} \mathscr{V} \text {. }
$$

The notion of a quasi-convex sequence is a generalization of a convex sequence. This concept was introduced by A. N. Kolmogorov in 1923 (cf. [2, 6, 7, 9]). From the above relations, we find that Theorem 1 can be applied to any of the mentioned classes. 
The fifth case we want to investigate is the following condition:

$\left\{c_{n}\right\}$ belongs to the class $2 \mathscr{M}$, i.e., $\left\{c_{n}\right\}$ is a null sequence such that

(v) for some $\alpha \geqslant 0, c_{n} / n^{\alpha}$ and $c_{-n} / n^{\alpha}$ are monotonically decreasing as $n$ varies from 1 to $\infty$

(cf. [2]). The quasi-monotonicity of $\left\{c_{n}\right\}$ yields

$$
\left|\Delta c_{n}\right| \leqslant \Delta c_{n}+K \alpha \frac{c_{n}}{|n|} \text { for all } n \neq 0,
$$

where $K=\max \left(2,2^{\alpha}\right)$. This implies that

$$
\sum_{|k|=n}^{[\lambda n]}\left|\Delta c_{k}\right| \leqslant c_{n}+c_{-n}-c_{[\lambda n]+1}-c_{-[\lambda n]-1}+M\left(\frac{1}{n}+\log \lambda\right) \sup _{|k| \geqslant n}\left|c_{k}\right|,
$$

where $M=2 \alpha \max \left(2,2^{\alpha}\right)$. From the above inequality, we find that condition $(\Delta)$ holds for $m=1$.

The sixth case we want to investigate is the following condition:

$\sum_{|n|<\infty} c_{n} e^{i n t}$ is a lacunary series, i.e., $\lim _{|n| \rightarrow \infty} c_{n}=0$, and there exists a sequence of positive integers, say $\left\{n_{k}\right\}_{k=1}^{\infty}$, such that $\gamma \equiv \inf _{k} n_{k+1} / n_{k}>1$, and such that $c_{n}=0$ except perhaps for $n=0, \pm n_{k}(k=1,2, \ldots)$.

The notion of a lacunary series was introduced by Hadamard in the study of the 'over-convergence' problem of a power series (cf. [5, 8]). From the definition, we can easily obtain

$$
\sum_{|k|=n}^{[\lambda n]}\left|c_{k}\right| \leqslant \sup _{k \geqslant n}\left(\left|c_{k}\right|+\left|c_{-k}\right|\right)
$$

for all $n \geqslant 1$ and all $\lambda$ with $1<\lambda<\gamma$. So condition $(\Delta)$ holds.

The seventh case we want to investigate is the following condition:

$$
\left\{\Delta^{s} c_{n}\right\}_{n>0} \text { and }\left\{\Delta^{s} c_{-n}\right\}_{n>0} \text { are monotone sequences } \quad(s \geqslant 0) .
$$

It is clear that condition (vii) implies ( $\Delta)$. A special case of condition (vii) is as follows: $\left\{c_{n}\right\}$ is an even sequence with $c_{n}=1 /(2 \log n)$ for $n \geqslant 2$. For instance, one may consider the famous example $\sum_{n \geqslant 2} \cos n t / \log n$, which does not belong to any $L^{p}(T)$ for $1<p \leqslant \infty$. 
Proof OF TheOREM 1. Obviously, the first assertion of Theorem 1 is implied by the second assertion. Therefore, to prove Theorem 1, it suffices to establish the second assertion. Suppose that $S_{n}(t)$ converges uniformly on $E$. Since each $S_{n}(t)$ is bounded on $T,\left\{S_{n}(t)\right\}$ is uniformly bounded on $E$, and its limit function is also bounded on $E$. Following the proof of [3, Theorem 5.3.1], except for a minor modification, we see that $\sigma_{n}(t)$ converges uniformly on $E$. Conversely, assume that $\sigma_{n}(t)$ converges uniformly on $E$. We claim that $S_{n}(t)$ converges uniformly on $E$. For $\lambda>1$, write $\lambda_{n}$ instead of $[\lambda n]$. From the definitions of $S_{n}(t)$ and $\sigma_{n}(t)$, we may easily see that

(*) $S_{n}(t)-\sigma_{n}(t)=\frac{\lambda_{n}+1}{\lambda_{n}-n}\left\{\sigma_{\lambda_{n}}(t)-\sigma_{n}(t)\right\}-\sum_{|k|=n+1}^{\lambda_{n}} \frac{\lambda_{n}+1-|k|}{\lambda_{n}-n} c_{k} e^{i k t}$.

It is clear that, for $(\lambda-1) n \geqslant 2$, we have

$$
\left|\frac{\lambda_{n}+1}{\lambda_{n}-n}\left\{\sigma_{\lambda_{n}}(t)-\sigma_{n}(t)\right\}\right| \leqslant \frac{4 \lambda}{\lambda-1}\left|\sigma_{\lambda_{n}}(t)-\sigma_{n}(t)\right| .
$$

This implies that, for each fixed $\lambda>1$, we have

$$
\left|\frac{\lambda_{n}+1}{\lambda_{n}-n}\left\{\sigma_{\lambda_{n}}(t)-\sigma_{n}(t)\right\}\right| \rightarrow 0 \text { uniformly on } E \text { as } n \rightarrow \infty .
$$

Now, we want to estimate the sum on the right side of the equation (*). Let $E_{n}^{*}(t)$ be defined as in [2], i.e., for $n>0$, define

$$
E_{n}^{*}(t)=\frac{e^{i(n+1) t}}{2 i e^{i t / 2} \sin t / 2}=\sum_{k=0}^{n} e^{i k t}+\frac{1}{2 i e^{i t / 2} \sin t / 2},
$$

and define $E_{-n}^{*}(t)=E_{n}^{*}(-t)$. By using summation by parts, we get

$$
\begin{aligned}
\sum_{k=n+1}^{\lambda_{n}} \frac{\lambda_{n}+1-k}{\lambda_{n}-n} c_{k} e^{i k t} & =\sum_{k=n+1}^{\lambda_{n}} \frac{\lambda_{n}+1-k}{\lambda_{n}-n} c_{k}\left(E_{k}^{*}(t)-E_{k-1}^{*}(t)\right) \\
& =\sum_{k=n}^{\lambda_{n}-1} \frac{\lambda_{n}+1-k}{\lambda_{n}-n} \Delta c_{k} E_{k}^{*}(t)+\phi_{1}(t, n, \lambda),
\end{aligned}
$$

where

$\phi_{1}(t, n, \lambda)=\frac{1}{\lambda_{n}-n} \sum_{k=n}^{\lambda_{n}-1} c_{k+1} E_{k}^{*}(t)-\frac{\lambda_{n}+1-n}{\lambda_{n}-n} c_{n} E_{n}^{*}(t)+\frac{1}{\lambda_{n}-n} c_{\lambda_{n}} E_{\lambda_{n}}^{*}(t)$.

We have

$$
\left|\phi_{1}(t, n, \lambda)\right| \leqslant \frac{2}{|\sin t / 2|} \sup _{k \geqslant n}\left|c_{k}\right| \text { for } \lambda_{n} \geqslant n+1
$$


This implies that for each fixed $\lambda>1, \phi_{1}(t, n, \lambda)$ converges to 0 uniformly on $\delta \leqslant|t| \leqslant \pi$ as $n \rightarrow \infty$. We want to show that

$$
\begin{aligned}
\sum_{k=n}^{\lambda_{n}-1} & \frac{\lambda_{n}+1-k}{\lambda_{n}-n} \Delta c_{k} E_{k}^{*}(t) \\
\quad= & \frac{e^{i t / 2}}{2 i \sin t / 2} \sum_{k=n-1}^{\lambda_{n}-2} \frac{\lambda_{n}+1-k}{\lambda_{n}-n} \Delta^{2} c_{k} E_{k}^{*}(t)+\phi_{2}(t, n, \lambda)
\end{aligned}
$$

for some function $\phi_{2}(t, n, \lambda)$ with the following property: for each fixed $\lambda>1$, $\phi_{2}(t, n, \lambda)$ converges to 0 uniformly on $\delta \leqslant|t| \leqslant \pi$ as $n \rightarrow \infty$. By using summation by parts, we get

$$
\begin{aligned}
\sum_{k=n}^{\lambda_{n}-1} \frac{\lambda_{n}+1-k}{\lambda_{n}-n} \Delta c_{k} E_{k}^{*}(t) & =\frac{e^{i t / 2}}{2 i \sin t / 2} \sum_{k=n}^{\lambda_{n}-1} \frac{\lambda_{n}+1-k}{\lambda_{n}-n} \Delta c_{k} e^{i k t} \\
= & \frac{e^{i t / 2}}{2 i \sin t / 2} \sum_{k=n}^{\lambda_{n}-1} \frac{\lambda_{n}+1-k}{\lambda_{n}-n} \Delta c_{k}\left(E_{k}^{*}(t)-E_{k-1}^{*}(t)\right) \\
= & \frac{e^{i t / 2}}{2 i \sin t / 2} \sum_{k=n-1}^{\lambda_{n}-2} \frac{\lambda_{n}+1-k}{\lambda_{n}-n} \Delta^{2} c_{k} E_{k}^{*}(t)+\phi_{2}(t, n, \lambda),
\end{aligned}
$$

where

$$
\begin{aligned}
\phi_{2}(t, n, \lambda)=\frac{e^{i t / 2}}{2 i \sin t / 2} & \left\{\frac{1}{\lambda_{n}-n} \sum_{k=n-1}^{\lambda_{n}-2} \Delta c_{k+1} E_{k}^{*}(t)\right. \\
& \left.-\frac{\lambda_{n}+2-n}{\lambda_{n}-n} \Delta c_{n-1} E_{n-1}^{*}(t)+\frac{2}{\lambda_{n}-n} \Delta c_{\lambda_{n-1}-1} E_{\lambda_{n}-1}^{*}(t)\right\} .
\end{aligned}
$$

We have

$$
\left|\phi_{2}(t, n, \lambda)\right| \leqslant \frac{6}{|2 \sin t / 2|^{2}} \sup _{k \geqslant n-1}\left|\Delta c_{k}\right| \text { for } \lambda_{n} \geqslant n+1 .
$$

This implies that for each fixed $\lambda>1, \phi_{2}(t, n, \lambda)$ converges to 0 uniformly on $\delta \leqslant|t| \leqslant \pi$ as $n \rightarrow \infty$. If we repeat the above procedure several times, we get, finally:

$$
\begin{aligned}
& \sum_{k=n+1}^{\lambda_{n}} \frac{\lambda_{n}+1-k}{\lambda_{n}-n} c_{k} e^{i k t} \\
& \quad=\left(\frac{e^{i t / 2}}{2 i \sin t / 2}\right)^{m-1} \sum_{k=n-m+1}^{\lambda_{n}-m} \frac{\lambda_{n}+1-k}{\lambda_{n}-n} \Delta^{m} c_{k} E_{k}^{*}(t)+\phi(t, n, \lambda),
\end{aligned}
$$

where $\phi(t, n, \lambda)$ is of the form

$$
\begin{aligned}
\phi(t, n, \lambda)= & \phi_{1}(t, n, \lambda)+\phi_{2}(t, n, \lambda) \\
& +\phi_{3}(t, n, \lambda)\left(\frac{e^{i t / 2}}{2 i \sin t / 2}\right)+\cdots+\phi_{m}(t, n, \lambda)\left(\frac{e^{i t / 2}}{2 i \sin t / 2}\right)^{m-2},
\end{aligned}
$$


and where each $\phi_{j}(t, n, \lambda)$ has the property: for any fixed $\lambda>1, \phi_{j}(t, n, \lambda)$ converges to 0 uniformly on $\delta \leqslant|t| \leqslant \pi$ as $n \rightarrow \infty$. It is clear that $\phi(t, n, \lambda)$ has the same property as $\phi_{j}(t, n, \lambda)$, i.e., for any fixed $\lambda>1, \phi(t, n, \lambda)$ converges to 0 uniformly on $\delta \leqslant|t| \leqslant \pi$ as $n \rightarrow \infty$. By the same argument as above, we can show that

$$
\begin{aligned}
& \sum_{k=n+1}^{\lambda_{n}} \frac{\lambda_{n}+1-k}{\lambda_{n}-n} c_{-k} e^{-i k t} \\
& \quad=\left(\frac{e^{-i t / 2}}{-2 i \sin t / 2}\right)^{m-1} \sum_{k=n-m+1}^{\lambda_{n}-m} \frac{\lambda_{n}+1-k}{\lambda_{n}-n} \Delta^{m} c_{-k} E_{-k}^{*}(t)+\Psi(t, n, \lambda),
\end{aligned}
$$

where $\Psi(t, n, \lambda)$ has the following property: for any fixed $\lambda>1, \Psi(t, n, \lambda)$ converges to 0 uniformly on $\delta \leqslant|t| \leqslant \pi$ as $n \rightarrow \infty$. Combining the last two equations, we get

$$
\begin{aligned}
& \left|\sum_{|k|=n+1}^{\lambda_{n}} \frac{\lambda_{n}+1-|k|}{\lambda_{n}-n} c_{k} e^{i k t}\right| \\
& \leqslant \frac{m+1}{|2 \sin t / 2|^{m}} \sum_{|k|=n-m+1}^{\lambda_{n}-m}\left|\Delta^{m} c_{k}\right|+|\phi(t, n, \lambda)|+|\Psi(t, n, \lambda)| \\
& \leqslant \quad \frac{m+1}{|2 \sin t / 2|^{m}}\left(\sum_{|k|=n}^{\lambda_{n}}\left|\Delta^{m} c_{k}\right|+2 m \sup _{|k| \geqslant n-m+1}\left|\Delta^{m} c_{k}\right|\right) \\
& \quad+|\phi(t, n, \lambda)|+|\Psi(t, n, \lambda)| .
\end{aligned}
$$

Let $K(n, \lambda)$ be a constant, dependent on $n$ and $\lambda$ only, such that

$$
|\phi(t, n, \lambda)|+|\Psi(t, n, \lambda)| \leqslant K(n, \lambda) \text { for all } \delta \leqslant|t| \leqslant \pi \text {, }
$$

and such that

$$
\lim _{n \rightarrow \infty} K(n, \lambda)=0 \quad \text { for each fixed } \lambda>1
$$

Then

$$
\begin{aligned}
& \sup _{\delta \leqslant|t| \leqslant \pi}\left|\sum_{|k|=n+1}^{\lambda_{n}} \frac{\lambda_{n}+1-|k|}{\lambda_{n}-n} c_{k} e^{i k t}\right| \\
& \leqslant \frac{m+1}{|2 \sin \delta / 2|^{m}}\left(\sum_{|k|=n}^{\lambda_{n}}\left|\Delta^{m} c_{k}\right|+2 m \sup _{|k| \geqslant n-m+1}\left|\Delta^{m} c_{k}\right|\right)+K(n, \lambda) .
\end{aligned}
$$

By the hypotheses on $\left\{c_{n}\right\}$ and $K(n, \lambda)$, we see that

$$
\text { (***) } \quad \lim _{\lambda \downarrow 1} \varlimsup_{n \rightarrow \infty} \sup _{n \leqslant|t| \leqslant \pi}\left|\sum_{|k|=n+1}^{\lambda_{n}} \frac{\lambda_{n}+1-|k|}{\lambda_{n}-n} c_{k} e^{i k t}\right|=0 \text {. }
$$


From $(*),(* *)$ and $(* * *)$, we find that $S_{n}(t)$ converges uniformly on $E$. For $m=0$, it is easy to see from the above proof that the conditions on $t_{0}$ and $E$ can be eliminated.

With the help of Lebesgue's theorem and Fejerr's theorem, it is easy to see that Theorem 1 has the following consequence.

Corollary 2. Let $f \in L^{1}(T)$ with the condition

$$
\lim _{\lambda \downarrow 1} \varlimsup_{n \rightarrow \infty} \sum_{|k|=n}^{[\lambda n]}\left|\Delta^{m} \hat{f}(k)\right|=0
$$

for some nonnegative integer $m$. Then the following conclusions hold.

(1) $S_{n}\left(f, t_{0}\right)$ converges to $\breve{f}\left(t_{0}\right)$ for every value of $t_{0}$ satisfying $0<\left|t_{0}\right| \leqslant \pi$ and

$$
\lim _{h \rightarrow 0} \frac{1}{h} \int_{0}^{h}\left|\frac{f\left(t_{0}+\tau\right)+f\left(t_{0}-\tau\right)}{2}-\check{f}\left(t_{0}\right)\right| d \tau=0 .
$$

In particular, $S_{n}(f, t)$ converges to $f(t)$ almost everywhere.

(2) Let $E$ be any closed subinterval of $T$ bounded away from 0 . If $f$ is continuous on $E$, then $S_{n}(f, t)$ converges to $f(t)$ uniformly on $E$.

(3) Whenever $m=0$, the condition ' $0<\left|t_{0}\right| \leqslant \pi$ ' in (1) and the condition ' $E$ is bounded away from 0 ' in (2) can be eliminated.

REMARK. As explained in the remark after Theorem 1, we see that Corollary 2 can be applied to any of the cases (i) to (vii) stated there. Let us discuss a special case of (i) as follows. Suppose that $f$ is a function of bounded variation. It is a well-known fact that $n \hat{f}(n)=O(1)$ as $|n| \rightarrow \infty$. This implies that the condition on $\{\hat{f}(n)\}$ in Corollary 2 corresponding to $m=0$ holds for such a case. On the other hand, it is well known that for any point $t_{0}, f\left(t_{0}+0\right)$ and $f\left(t_{0}-0\right)$ exist, which implies that

$$
\lim _{h \rightarrow 0} \frac{1}{h} \int_{0}^{h}\left|\frac{f\left(t_{0}+\tau\right)+f\left(t_{0}-\tau\right)}{2}-\check{f}\left(t_{0}\right)\right| d \tau=0,
$$

where $\check{f}\left(t_{0}\right)=\frac{1}{2}\left\{f\left(t_{0}+0\right)+f\left(t_{0}-0\right)\right\}$. The above discussion tells us that Corollary 2 generalizes the Jordan test theorem, i.e., [8, Theorem 13.232] or [5, Corollary II.2.2]. From the inequality

$$
\sum_{n \leqslant|k| \leqslant 2 n}|\hat{f}(k)| \leqslant n^{-1} \sum_{|k| \leqslant 2 n}|k \hat{f}(k)|,
$$

we see that Corollary 2 also generalizes Fatou's theorem in [3, Vol. 1, page 106].

We have seen how the proof of Theorem 1 goes through for the case $\lambda_{n}=[\lambda n]$. Following the same proof, except for a minor modification, we can easily find that the proof also works for the case $\lambda_{n}=n+\left[n / l_{n}\right]$. This means that the following result is true. 
THEOREM 3. Let $\left\{c_{n}\right\}$ be a null sequence and let $0<\left|t_{0}\right| \leqslant \pi$. If there exists $a$ sequence, say $\left\{l_{n}\right\}_{n>0}$, such that

(1) $1 \leqslant l_{n} \leqslant n$ for all $n$,

(2) $l_{n}\left|\sigma_{n+\left[n / l_{n}\right]}\left(t_{0}\right)-\sigma_{n}\left(t_{0}\right)\right|=o(1)(n \rightarrow \infty)$, and

(3) $\lim _{n \rightarrow \infty} \sum_{|k|=n}^{n+\left[n / l_{n}\right]}\left|\Delta^{m} c_{k}\right|=0$ for some nonnegative integer $m$,

then $S_{n}\left(t_{0}\right)$ and $\sigma_{n}\left(t_{0}\right)$ converge together to the same limit, or they both diverge. Furthermore, suppose that condition (2) holds uniformly on $E$, where $E$ is bounded away from 0 . Then $S_{n}(t)$ converges uniformly on $E$ if and only if $\sigma_{n}(t)$ converges uniformly on $E$. Whenever $m=0$, the conditions on $t_{0}$ and $E$ can be eliminated.

In [2], the $L^{1}$-convergence property of a Fourier series satisfying one of the following conditions was established:

$$
\begin{gathered}
l_{n}^{-1 / q}\left(\sum_{|k|=n}^{n+\left[n / l_{n}\right]}|k|^{p-1}\left|\Delta c_{k}\right|^{p}\right)^{1 / p}=o(1) \quad(n \rightarrow \infty), \\
\left(\log \left[n / l_{n}\right]\right) \sum_{|k|=n}^{n+\left[n / l_{n}\right]}\left|\Delta c_{k}\right|=o(1) \quad(n \rightarrow \infty),
\end{gathered}
$$

where $1<p \leqslant 2,1 / p+1 / q=1$ and $c_{n}=\hat{f}(n)$ for all $n$. Obviously, condition (iii') implies condition (3) in Theorem 3 corresponding to $m=1$. On the other hand, by the Hölder inequality, we get

$$
\sum_{|k|=n}^{n+\left[n / l_{n}\right]}\left|\Delta c_{k}\right| \leqslant\left(\frac{4}{l_{n}}\right)^{1 / q}\left(\sum_{|k|=n}^{n+\left[n / l_{n}\right]}|k|^{p-1}\left|\Delta c_{k}\right|^{p}\right)^{1 / p},
$$

where $1<p \leqslant 2$ and $1 / p+1 / q=1$, which says that condition (ii') also implies the same condition. In conclusion, Theorem 3 complements the results on pointwise convergence of these Fourier series. It is obvious that Theorem 3 is better than Theorem 1 for the case that the sequence $\left\{l_{n}\right\}$ exists. From the fact that

and that

$$
\left\|\sigma_{n}(f)-f\right\|_{\infty}=O\left(n^{-\alpha}\right) \quad \text { for } f \in \operatorname{Lip}_{\alpha}(T), 0<\alpha<1
$$

$$
\left\|\boldsymbol{o}_{n}(f)-f\right\|_{\infty}=O(\log n / n) \quad \text { for } f \in \operatorname{Lip}_{1}(T),
$$

we know that the sequence $\left\{l_{n}\right\}$ exists at least for functions satisfying a Lipschitz condition. This shows that Theorem 3 makes sense at least for Lipschitz classes. From the definition of $\left\{l_{n}\right\}$, we know that the existence problem of the sequence $\left\{l_{n}\right\}$ is completely dependent on the estimate of the quantity $\left|\sigma_{\lambda_{n}}\left(t_{0}\right)-\sigma_{n}\left(t_{0}\right)\right|$. Therefore, obtaining a better estimate of the quantity $\left|\sigma_{\lambda_{n}}\left(t_{0}\right)-\sigma_{n}\left(t_{0}\right)\right|$ is a problem of special significance. 


\section{Acknowledgement}

I want to take this opportunity to express my gratitude to Professor Paul J. Cohen for his help in the preparation of this paper. I would also like to thank the referee for his valuable advice in developing the final version of this paper.

\section{References}

[1] R. Bojanic and Č. V. Stanojević, 'A class of $L^{1}$-convergence', Trans. Amer. Math. Soc. 269 (1982), 677-683.

[2] C.-P. Chen, ' $L^{1}$-convergence of Fourier series,' J. Austral. Math. Soc. (Series A) 41 (1986), $376-390$.

[3] R. E. Edwards, Fourier Series. A Modern Introduction, Vols. 1 and 2 (Holt, Rinehart and Winston, New York, 1967).

[4] J. Karamata, Teorija i praksa Stieltjes-ova integrala (Srpska Akademija Nauka, Beograd, 1949).

[5] Y. Katznelson, An Introduction to Harmonic Analysis (John Wiley and Sons, New York, 1968).

[6] A. N. Kolmogorov, 'Sur l'ordre de grandeur des coefficients de la série de Fourier-Lebesgue', Bull. Acad. Polon. Sci. Sér. Sci. Math. Astronom. Phys. (1923), 83-86.

[7] Č. V. Stanojević, 'Classes of $L^{1}$-convergence of Fourier and Fourier-Stieltjes series,' Proc. Amer. Math. Soc. 82 (1981), 209-215.

[8] E. C. Titchmarsh, Theory of Functions (2nd ed., Oxford University Press, 1958).

[9] A. Zygmund, Trigonometric Series (Cambridge Univ. Press, 1959).

Department of Mathematics

Stanford University

Stanford, California 94305

U.S.A. 